\title{
La formation éthique des ingénieurs en France : une naissance difficile
}

\section{Christelle Didier}

\section{(2) OpenEdition \\ Journals}

Édition électronique

URL : http://journals.openedition.org/trema/3635

DOI : $10.4000 /$ trema.3635

ISSN : 2107-0997

Éditeur

Faculté d'Éducation de l'université de Montpellier

Édition imprimée

Date de publication : 1 décembre 2017

Pagination : 05-15

ISBN : 979-10-96627-03-5

ISSN : 1167-315X

Référence électronique

Christelle Didier, «La formation éthique des ingénieurs en France : une naissance difficile », Tréma [En ligne], 47 | 2017, mis en ligne le 01 février 2018, consulté le 19 avril 2019. URL : http:// journals.openedition.org/trema/3635 ; DOI : 10.4000/trema.3635

Ce document a été généré automatiquement le 19 avril 2019.

Trema 


\title{
La formation éthique des ingénieurs en France : une naissance difficile
}

\author{
Christelle Didier
}

\section{Introduction}

1 L'éthique est « à la mode » depuis les années 1990 après avoir été un champ déserté par la recherche, comme l'ont montré les rares sociologues intéressés par la question (Terrenoire, 1991 ; Isambert, Ladrière, et Terrenoire, 1978). Qu'il s'agisse d'un retour à " l'inévitable morale » dans une société bousculée par des transformations complexes (Valadier, 1991), un effet de mode passager, une « valse » (Etchegoyen, 1991) ou encore la quête d'une " éthique indolore " (Lipovetsky, 1992), aucune sphère de la vie et aucune profession ne semblent échapper à la question. Les médecins et les biologistes ont été les premiers touchés, mais, ils ne sont plus les seuls concernés : économistes, politiques, enseignants, travailleurs sociaux et journalistes sont sommés de répondre de leurs pratiques devant leurs contemporains et de redéfinir leurs responsabilités sociales et sociétales.

2 L'intérêt pour l'éthique en lien avec les activités professionnelles n'est pas nouveau: Emile Durkheim appelait déjà de ses vœux la fondation de morales professionnelles permettant de fédérer les membres d'une même « industrie » et les prémunir des risques d'anomie (Durkheim, 1967 [1893]). On parle aujourd'hui d'éthique professionnelle plutôt que de morale professionnelle sans que la distinction entre les deux expressions ne soit toujours claires, ni forcément nécessaire (Kahn, 2006). Quoi qu'il en soit, cet intérêt connaît des formes d'institutionnalisation diverses : codes de déontologie sans statut légal ni pouvoir de coercition ou ayant force de loi ; chartes ou code promulgués par des organisations publiques ou privées aux statuts variés ; comité d'éthique souvent consultatifs...

3 Des formations en éthique professionnelle se sont développées depuis les années 1970, d'abord dans le secteur médical et d'abord aux Etats-Unis. Elles ont commencé à susciter de l'intérêt en France dans les années 1990 dans le champ de la santé. Dans les études de 
médecine, l'éthique est un des objectifs généraux pour les sciences humaines et sociales, évaluées depuis 2009 en fin de première année. Dans les écoles d'ingénieurs, la formation éthique est encouragée par l'organisme d'accréditation, la Commission des titres d'ingénieurs (CTI) depuis plus de vingt ans. Mais les cours sont encore rares, de même que les recherches à leur sujet.

L'article présenté ici vise à comprendre le lent développement de la formation éthique des ingénieurs en France. Dans un premier temps, nous évoquerons la position de la CTI et ferons un point sur l'état de la recherche en éducation sur le sujet. Dans une perspective plus centrée sur la nature des connaissances qui caractérise l'éthique professionnelle, nous mettrons à jour des dynamiques d'institutionnalisation observées dans d'autres pays. Une comparaison avec l'éthique médicale, qui y est devenue un champ légitime de recherche et d'enseignement en France, nous permettra de mettre à jour quelques spécificités liées au domaine de l'ingénierie dans le contexte français.

\section{Un état des lieux difficile}

\section{1. L'attention de la CTI}

5 Les élèves-ingénieurs poursuivent en France une formation de cinq ans qui comprend surtout des apports dans les domaines scientifiques et techniques, en gestion et marketing et des mises en situation professionnelle proposées surtout les trois dernières années. La philosophie et l'épistémologie ont trouvé leur place dans certaines écoles depuis longtemps (Galiacy et Havelange, 1983) mais les enseignements relevant des SHS et/ou de "l'autre formation des ingénieurs» (Derouet, 2010) ne se sont diffusés que récemment (Lemaître, 2003) (Roby, 2014). C'est dans cet ensemble à l'intitulé encore instable (formation humaine, sociale, générale, non technique ; sciences humaines et sociale (SHS) ou encore SHS "pour l'ingénieur») et qui accueille des contenus aussi divers que le sport, le théâtre et les langues étrangères, que l'éthique a trouvé sa place.

6 La CTI qui constitue la pierre angulaire de la formation des ingénieurs français depuis 1934 a commencé à citer explicitement l'éthique comme objectif pour la formation générale en 1995. C'est d'abord une "ouverture à la réflexion éthique sur le métier » (CTI, 1995) qui a été évoquée renvoyant à une acception déontologique, entre la morale professionnelle de Durkheim et l'ethics, comprise comme un attribut essentiel des professions dans la théorie fonctionnaliste (Parsons, 1968) (Dubar, Tripier, et Boussard, 2015 [1998]). Plus tard, la CTI a évoqué une "ouverture à la réflexion éthique » sans référence au métier (CTI, 2000), induisant un sens du mot éthique moins strictement déontologique, mais aussi moins précis, et loin des usages en cours dans les champs émergents de l'éthique dite professionnelle ou appliquée, ou encore sectorielle (Durand, 2005, p. 144).

7 Bien que réitérée à chaque réécriture du référentiel, l'invitation de la CTI n'a pas suffi à généraliser la formation éthique dans les écoles d'ingénieurs. La Commission a poursuivi en élargissant même ses attentes. La dernière version d'Analyse et perspectives incite en effet « chaque école à se pencher sur ce sujet et à préciser la démarche qu'elle souhaite mettre en œuvre sur les trois axes d'analyse: la place de l'éthique dans le métier de l'ingénieur, la place de l'éthique dans la formation des ingénieurs, la place de l'éthique dans la conduite d'une école d'ingénieur » (CTI, 2014). Si l'intention de développer la place de l'éthique dans la formation des ingénieurs est là, les récents audits de la CTI 
évoquent davantage les SHS en général que l'éthique, peu citée dans les conclusions des rapports.

\section{2. L'éthique dans les recherches en éducation}

8 Malgré l'absence de cadre de la part de la CTI sur ce que devrait ou pourrait être un curriculum en éthique, des cours ont été créés. Certains même avant 1995 : un premier donné dans un MBA lillois destiné à des ingénieurs a été suivi de quelques autres dans le Nord de la France conçus par une petite équipe d'enseignants soutenue par l'Institut catholique de Lille. L'initiateur du Centre d'éthique technologique créé en 1993 avait découvert le sujet Outre-Atlantique (Didier, 1993) (Hériard Dubreuil, 1997 ; Didier, Gireaux-Geneau et Hériard Dubreuil, 1998). La publication des Références et orientations de la CTI en 1995 a encouragé la création de nouveaux cours, d'abord confiés à des vacataires (professionnels et enseignants du secondaire), puis plus souvent à des permanents et des enseignants-chercheurs des écoles.

9 L'ampleur de ce déploiement est difficile à évaluer car les connaissances issues de la recherche sont dispersées dans des travaux sur les contenus "non techniques» des formations (Lemaître, 2003) (Albero et Roby, 2014) ou sur les valeurs et idéologies de la profession où la formation est abordée marginalement (Didier, 2008). On trouve dans la littérature des études sur le cas de l'école des Mines de Douai (Triffaux, 1997) (Didier 2000), sur l'utilisation pédagogique des controverses scientifiques et techniques (Vinck, 2007), la place du développement durable et de la Responsabilité Sociale des Entreprises (RSE) (Didier et Huët, 2008), contenus qui cherchent leur place entre formation professionnelle et "éducation a " (Prévost et Jouffray, 2013) pas toujours identifiés comme relevant de l'éthique.

10 La formation des ingénieurs fait l'objet d'un intérêt croissant en sciences de l'éducation depuis une dizaine d'années (Sonntag, Lemaître, Fraysse, Becerril, et Oget, 2008), complétant l'approche socio-historique initiée par André Grelon. Ces recherches, souvent menées par des maîtres de conférences recrutés dans les écoles, prolongent un intérêt ancien de la CTI, du Centre d'Etude sur les Formations et l'emploi des Ingénieurs (CEFI) ${ }^{1}$ ainsi que d'associations et syndicats d'ingénieurs (Derouet et Paye, 2010).

11 La thèse pionnière de Annie Dufour étudiait les différentes conceptions de l'enseignement de la sociologie dans une école d'ingénieur agricole (Dufour, 1998), celle de Gilles Lazuech analysait en sociologue les "formations en compétences étrangères » dans le cadre de l'internationalisation des écoles (Lazuech, 1998). Denis Lemaître a identifié trois modèles de formation " humaine »: humanités, centrées sur la transmission d'une culture littéraire, philosophique, artistique ; développement personnel, centré sur des activités visant l'épanouissement ; et sciences sociales pour l'ingénieur, centrées sur l'adaptation aux contextes professionnels (Lemaître, 2001). Christelle Didier a identifié deux ancrages des formations « non-techniques »: universaliste où prime la culture générale, dans les écoles d'élites et social, développé dans les écoles confessionnelles et leur objectif réformiste de développer le " rôle social de l'ingénieur » (Didier, 2002). Catherine Roby a identifié une interdépendance entre les affichages de SHS des écoles et leurs orientations culturelles technoscientifiques (Roby, 2014).

12 Le développement de la recherche sur les formations d'ingénieurs et en particulier sur les SHS s'est accompagné d'une institutionnalisation de la recherche en SHS dans les écoles. Quelques jalons sont à signaler: un numéro de Recherche et Formation sur les SHS en 
écoles d'ingénieur (Chaix et Bardel-Denonain, 1998), la création du colloque Question de pédagogie dans l'enseignement supérieur puis du réseau de recherche ingénium qui rassemble aujourd'hui plus de 200 enseignants-chercheurs en SHS exerçant en école d'ingénieurs et dont certains ont participé à la rédaction du texte « dimension humaine, économique et sociale » de la CTI, ainsi que les travaux menés de longue date à l'INSA de Lyon, organisateur du premier colloque international sur « l'éthique de l'ingénieur » en France en 1992 (Didier, 2008), comme l'ouvrage collectif dirigé par Michel Faucheux et Joëlle Forest (2007). Les thèses pionnières et ces travaux collectifs ont surtout mis en évidence le faible degré de consensus sur les objectifs de l'" autre formation » des ingénieurs et peu abordé l'éthique.

\section{Deux modèles de développement}

\section{1. De la déontologie à l'éthique, en Amérique du Nord}

13 Aux Etats-Unis, la formation éthique des ingénieurs s'est développée dans un contexte où préexistait une déontologie produite par une dynamique de professionnalisation (Layton, 1971). Elle a été encouragée à la fin des années 70 par la National Science Foundation qui a soutenu la publication des premiers manuels d'éthique pour ingénieurs (Mitcham, 1993). Elle a aussi bénéficié de l'exigence formulée par l'Accreditation Board of Engineering and Technology (ABET), équivalent américain de la CTI, de mettre en place pour l'an 2000 des formations éthiques pour tous les ingénieurs. Cette demande suivie de contrôles devenus plus exigeants a contribué à légitimer et structurer le champ.

Les cours proposés aux Etats-Unis comportent surtout des études de cas d'accidents ou incidents ayant donné (ou qui aurait dû donner) lieu à des divulgations (whistleblowing) et l'étude de codes de déontologie (Colby et Sullivan, 2008). L'approche philosophique d'abord dominante des premiers binômes ingénieurs/philosophes (philosophie des professions et philosophie analytique) (Schaub et Pavlovic, 1983) (Martin et Schinzinger, 1983) (Harris, Pritchard, et Rabins, 1995), s'est diversifiée avec l'intervention de chercheurs en Science and Technology Studies (STS) donnant des cours sur la justice sociale, la paix, la méritocratie, les questions de genre - toujours en lien avec le métier - ou encore les rapports entre technique et politique (Mitcham, 2015). Il existe aux Etats-Unis des (full ) Professors of Engineering Ethics, souvent à la fois docteur en ingénierie et/ou ingénieur (parfois Professional Engineer) et docteur en philosophie, SHS ou STS.

$\mathrm{Au}$ Québec, la formation éthique a été longtemps strictement juridique et déontologique : l'adhésion à l'Ordre des Ingénieurs du Québec (OIQ), nécessaire pour pouvoir exercer, repose sur la réussite d'un examen professionnel sur le droit et la déontologie, passé en dehors de l'université. Ce n'est qu'en 2004 que l'OIQ a mandaté un groupe de travail pour penser " l'intégration du professionnalisme et de l'éthique dans la formation des ingénieurs » (Begin, 2006), quinze après la parution du premier manuel d'éthique dédié aux futurs ingénieurs (Racine, Légault, et Begin, 1991). En 2007, Georges Legault identifiait quatre approches de la formation " humaniste ": socioculturelle, communicationnelle, déontologique et éthique. Il distinguait nettement l'apprentissage du raisonnement déontologique par lequel « on peut déterminer si le comportement envisagé est conforme à la norme légale ou morale » de l'apprentissage du raisonnement éthique permettant " d'évaluer un plan d'action afin de déterminer le meilleur choix possible » (Legault, 2007). En 2014 la Confédération pour le rayonnement étudiant en 
ingénierie au Québec (CRÉIQ) signalait les lacunes de la formation éthique des ingénieurs dans un mémoire présenté à la Commission Charbonneau qui a siégé de 2011 à 2014 suite aux affaires de corruption qui ont fait scandale dans le milieu de l'ingénierie au Québec dans les années 2000 (Larochelle, 2013). Les auteurs y suggéraient une vaste réforme des enseignements, le recours au conseil de chercheurs en éthique appliquée et des objectifs visant davantage la formation de l'esprit critique (CREI-CESO, 2014).

\section{2. Une éthique philosophique et critique au Pays-Bas}

Les Pays Bas avec ses $25 \%$ de surface en dessous du niveau de la mer est un pays qui dépend des ingénieurs. C'est aussi un pays où les STS sont passées d'une activité marginale pour les enseignants-chercheurs à un champ institutionnalisé avec de nombreux programmes doctoraux (Brumsen, 2005). C'est aussi un pays où la recherche en philosophie de l'ingénierie et des techniques fait référence à l'échelle internationale (Mitcham, 2015) avec son laboratoire 4.TU Ethics qui fédère les départements de philosophie des universités technologiques et agronomiques du pays. Plus de soixante chercheurs y travaillent sur l'éthique de la conception (design ethics), les big datas, l'innovation frugale, les méthodologies d'intervention éthique en recherche et développement, le rôle des émotions dans les innovations (http:// ethicsandtechnology.eu/).

L'institutionnalisation académique de l'éthique n'y repose pas sur la préexistence d'une déontologie, mais sur une volonté politique, celle de la loi sur l'enseignement supérieur de 1993 qui a fait de la responsabilité social un objectif de formation de l'université. Un groupe de travail de l'Université Technologique de Delft suggéra dès 1994 de rendre obligatoire la formation éthique dans tous les cursus et formula des objectifs pédagogiques : en 1996, les 10000 étudiants des huit facultés de Delft ont suivi ce cours (Van de Poel, Zandvoort, et Brumsen, 2001). D’abord fondés sur les méthodes et manuels états-uniens, les cours d'éthique de Delft ont progressivement laissé place à des enseignements construits sur des recherches originales. Depuis 2011, un manuel néerlandais (Van de Poel et Royakkers, 2011) offre en anglais, pour une audience internationale, une alternative aux manuels états-uniens.

\section{Une éthique de l'ingénierie en France}

\section{1. Des pressions insuffisantes}

En France, pays trois fois plus peuplé que les Pays-Bas et doté de formations d'ingénieurs nombreuses, anciennes et prestigieuses, il n'existe ni laboratoire ni chaire professorale dédiés à l'éthique ; le premier manuel français a trouvé son éditeur en Belgique (Didier, Hériard Dubreuil, et Gireaux-Geneau, 1998). La sollicitation ancienne de la CTI au sujet de la formation éthique ne s'est pas accompagnée comme pour les SHS d'un texte-cadre, juste d'annexes « déontologiques »: la Charte de 2001 de l'association des Ingénieurs et Scientifiques de France (IESF), le serment d'Archimède de l'Ecole Polytechnique Fédérale de Lausanne (1990), et celui de l'Institut national et polytechnique de Grenoble (2006). Ce cadrage manque d'autant plus que la formation éthique se trouve toujours à la marge, voire en dehors, des disciplines des enseignants sollicités, quand elle n'est pas confiée à 
des experts auto-proclamés. C'est la situation que décrivait Michiel Brumsen au sujet des STS au Pays-Bas dans les années 1970, situation bien dépassée vingt ans plus tard.

Une des explications du « retard » de la France repose sur l'organisation particulière de la profession. La longue absence de référence déontologique pour les ingénieurs français s'explique sans doute par son modèle d'organisation collective : l'adhésion à l'association qui les représente est facultative et indirecte, les adhérents sont surtout les associations d'anciens (Didier, 1999). Mais nous savons mieux aujourd'hui que le modèle états-unien est culturellement marqué, tout comme celui du Québec. Nous savons surtout que la formation éthique ne dépend pas de la préexistence d'une déontologie formelle, quel que soit son statut au regard de la loi. Elle dépend avant tout d'une volonté politique qui dépasse les intentions et soutienne la constitution d'un champ d'expertise. Le cas des Pays-Bas montre que l'éthique de l'ingénierie peut très bien se développer en l'absence de code. Sans creuser plus avant le cas Allemand traité par ailleurs (Huning et Mitcham, 1993), notons que le développement de l'éthique de l'ingénierie s'y est inscrit dans une culture où la philosophie s'intéressait depuis longtemps à la technique et où la crise morale traversée par la profession lors de la Seconde guerre mondiale a été déterminante : la réflexion sur l'impact des technologies y est aujourd'hui considérée comme une compétence incontournable des ingénieurs (Ropohl, 2001) (Downey, Lucena, et Mitcham, 2007).

\section{2. Ethique et professionnalité}

20 Le développement de l'éthique de l'ingénierie ne dépend donc pas tant de la préexistence d'une déontologie elle-même liée aux modalités d'organisation de la profession de son contexte juridique et culturel singulier. Il dépend en revanche fortement de l'existence d'une volonté politique. La comparaison avec le champ de la santé permet d'identifier un autre facteur, lié à l'évolution historique des pratiques et la façon qu'ont les praticiens d'en appréhender les enjeux éthiques.

21 Dans le domaine de la santé, la transformation des pathologies et la spécialisation de plus en plus poussée des acteurs ont conduit les médecins à travailler davantage avec d'autres professionnels. Le contexte de raréfaction des ressources et l'émergence de nouveaux droits pour les patient ont aussi modifié la façon de penser le métier et fait de l'éthique une composante majeure de la professionnalité (Ullerne-Weité, 2007) (Monceau, 2006). La création de formations recentrées sur les pratiques cliniques et la gestion des problèmes complexes de soins dans des diplômes universitaires et interuniversitaires, des Masters, ainsi que dans les « espaces éthique » institués par la loi de 2004 relative à la politique de santé témoignent de ces évolutions.

Comme pour les médecins, le travail des ingénieurs est de plus en plus spécialisé et s'accompagne d'une remise en question de l'expertise qui se traduit ici par une demande de transparence plus grande des choix techniques. Les ingénieurs doivent aussi faire face à des enjeux économiques liés à la mondialisation des marchés. Des cours ont été créés qui doivent répondre aux nouveaux besoins sociétaux, aux attentes des futurs employeurs, mais aussi permettre aux écoles de se distinguer (Lazuech, 1998). L'absence d'une pression claire de la part de la CTI, prescripteur légitime, est aussi le reflet de ces injonctions contradictoires dans un contexte national où les ingénieurs ne souffrent ni d'un déficit de reconnaissance sociale (États-Unis), ni de la défiance du public (Québec). 
Ils ne vivent pas dans un pays qui compte de façon vitale sur de leur ingéniosité (PaysBas) et n'ont pas traversé de crise existentielle (Allemagne).

Gardons enfin à l'esprit le contexte de l'enseignement supérieur tiraillé partout dans le monde entre académisation et professionnalisation (Lemaître, 2011). Comme pour les médecins, la valeur professionnelle du diplôme d'ingénieur repose sur d'autres axes de formation que les seules compétences technico-scientifiques (Bot 2007), d'où la place accordée aux SHS dès la première des formations médicales et dans les écoles d'ingénieurs. Mais l'éthique n'est pas perçue en France comme une discipline universitaire, en dehors de l'interstice accordé à l'éthique médicale (Thiel, 2014).

Un espace académique où se confronteraient ingénieurs et chercheurs est difficilement pensable dans le contexte où les ingénieurs n'ont pas la légitimité académique des médecins.

\section{Conclusion}

Des raisons culturelles et historiques expliquent la différence d'ampleur et de forme que prend le souci de l'éthique professionnelle selon les contextes nationaux. Si le développement de l'enseignement de l'éthique de l'ingénierie constitue dans certains pays pionniers un dépassement ou un déplacement d'une formation (souvent hors université) centrée sur la déontologie, il est issu aux Pays-Bas par exemple d'une volonté politique de développer la responsabilité sociale des étudiants. En France, la formation éthique des ingénieurs ne provient ni de l'évolution d'une formation déontologique ni d'une volonté politique forte. Elle bute sur la difficulté à établir un champ expert dédié à cette question, qu'ont connues les STS puis l'éthique aux Pays-Bas avant de s'institutionnaliser, que connait encore l'éthique médicale en France. Notre dernier détour par la comparaison avec le champ de la médecine a également mis en évidence des conceptions de la professionnalité variant selon les deux métiers. Tandis que l'éthique semble devenir une dimension intrinsèque du professionnalisme, pour les médecins, ce n'est pas le cas pour les ingénieurs, en tout cas en France. L'éthique de l'ingénierie reste un bricolage, un questionnement au croisement de la philosophie et des sciences humaines venant interroger la pratique de l'extérieur. L'éthique médicale qui est passée d'une sous-discipline de la philosophie à un champ atypique, interdisciplinaire et hybridé de théories émanant de la pratique, indépendant du travail propre de la déontologie, pourrait servir de modèle. Mais le chemin est long vers la constitution d'une ressource interne pour un professionnalisme " ingéniérial " qui serait le pendant du professionnalisme médical. Peut-être faudrait-il commencer par construire le champ d'expertise de l'éthique, hors de chacun de ses secteurs spécifiques, par la recherche. Selon un rapport remis au Sénat en 2013, la garantie de proposer des formations solides en éthique devrait passer par la reconnaissance de l'éthique pour elle-même à travers la constitution d'une section interdisciplinaire par la Commission nationale des universités sur le modèle par exemple des sciences de l'éducation. À méditer. 


\section{BIBLIOGRAPHIE}

Albero, B., et Roby, C. (2014). Les enjeux du rapport aux sciences humaines et sociales dans les formations d'ingénieurs. Revue Française de Pédagogie, 186, 59-73. DOI : 10.4000/rfp.4405

Begin, L. (2006). Professionalisme et éthique dans la formation des futurs ingénieurs au Québec. Conférence générale OCDE/IMHE "Value and Ethics: Managing Challenges and Realities in Higher Education". Paris : OCDE/IMHE. https://www.oecd.org/fr/sites/imhe2006bis/37244792.pdf Bordé, J., Delerue, N., et Suzor-Weiner, A. (2015). Pugwash : les physiciens, l'arme nucléaire, la responsabilité des scientifiques. Reflets physiques, 43, 51-53. DOI : 10.1051/refdp/201543051

Bot, L. (2007). Eléments d'une crise post-moderne dans la formation des ingénieurs. Les sciences de l'éducation - Pour l'ère nouvelle, 40 (3), 41-57. DOI : 10.3917/lsdle.403.0031

Brumsen, M. (2005). Ethics in Engineering in the Netherlands. The Role of Professional Association, Universities and Law. International Journal of Engineering Education, 21(3), 391-401.

Cadieux, N., et Laflamme, R. (2009). Ethique professionnelle et éthique en ingénierie. Relations industrielles, 64 (2), 307-325. DOI : 10.7202/037923ar

Chaix, M.-L., et Bardel-Denonain, O. (dir.) (1998). Les sciences humaines et sociales dans les formations d'ingénieurs. Recherche et Formation, 29. http://www.persee.fr/issue/ refor_0988-1824_1998_num1

Colby, A., et Sullivan, W. (2008). Ethics Teaching in Undergraduate in Engineering Education. Journal of Engineering Ethics, 93 (3), 327-333. DOI : 10.1002/j.2168-9830.2008.tb00982.x

CREI-CESO. (2014). Mémoire portant sur l'enseignement de l'éthique dans les programmes d'ingénierie au Québec. Montréal : CREIQ.

CTI. (1995). Références et orientations. Paris : CTI.

CTI. (2000). Références et orientations. Paris : CTI.

Davis, M. (1998). Thinking like an Engineer. Study in the Ethics of a Profession. Oxford : Oxford University Press.

Derouet, A. (2010). L'invention de l'autre formation des ingénieurs : débats et controverses autour des enseignements non techniques au milieu du XX siècle. Les cahiers du RECITS, 7.

Derouet, A., et Paye, S. (2010). Quand les ingénieurs débattent de leur formation non technique. Paris : CGC-CFE.

Derouet, A. (2015). La composition du corps professoral comme reflet du rapport des écoles d'ingénieurs $\mathrm{au}(\mathrm{x})$ monde(s) économique (s). Formation emploi, 132, 33-50. URL : http:// formationemploi.revues.org/4540

Derouet, A., et Thivet, D. (2015). L'enseignement juridique comme socialisation à une élite économique : le cas de l'Ecole centrale de Paris. Les cahiers de la recherche sur l'éducation et les savoirs, 14, 47-67. https://cres.revues.org/2751.

Didier, C. (1993). Une éducation morale des ingénieurs est-elle possible? Mémoire de Maîtrise en sciences de l'éducation.Villeneuve d'Ascq : Université Charles de Gaulle. 
Didier, C. (1999). Engineering Ethics in France : A Historical Perspective. Technology in Society, 471-486. DOI : 10.1016/S0160-791X(99)00029-9

Didier, C. (2002). Ethique et identités professionnelles des ingénieurs. Thèse de doctorat en sociologie. Paris : EHESS.

Didier, C. (2008). Penser l'éthique des ingénieurs. Paris : Presses Universitaires de France.

Didier, C. (2009). Engineering Ethics. Dans B. O. J.K., S. Pedersen, and H.V. (dir), A Companion to the Philosophy of Technology (pp.427-432). Oxford : Wiley-Blackwell.

Didier, C. (2010). Les ingénieurs et l'éthique professionnelle. Pour une approche comparative de la déontologie. Dans D. Demazière, et $\mathrm{C}$. Gadéa, Sociologie des groupes professionnels. Acquis récents et nouveaux défis (pp. 208-218). Paris : La Découverte.

Didier, C. (2015). Engineering Ethics, A European Perspective. Dans J. Britt Holbrook, and 2. ed. (Éd.), Ethics, Science,Technology and Engineering : A Global Resource (Vol. 2, pp. 87-90). Farmington Hills, MI : Macmillan reference USA.

Didier, C. (2015). Foreword. Dans S. Sundar Sethy, Contemporary Ethical Issues in Engineering (pp. xvi-xxi). Hershey, PA : IGI Global.

Didier, C., Hériard Dubreuil, B., et Gireaux-Geneau, A. (1998). Ethique industrielle.Textes pour un débat. Bruxelles : De Boeck université.

Didier, C., et Huët, R. (2008). Corporate Social Responsibility in Engineering Education. A French Survey. European Journal of Engineering Education, 33 (2), 169-177. DOI : 10.1080/03043790801976472

Didier, C., et Derouet, A. (2011). Social Responsibility in French Engineering Education : A Historical and Sociological Analysis. Science and Engineering Ethics, 19 (4), 1577-1588. DOI : 10.1007/ s11948-011-9340-9

Downey, G. L., Lucena, J., and Mitcham, C. (2007). Engineering Ethics and Identity : Engineering Initiative in Comparative Perspective. Science and Engineering Ethics, 13, 463-487. DOI :

10.1007/978-3-319-16172-3_5

Dubar, C.,Tripier, P., et Boussard,V. (2015 [1998]). Sociologie des professions (éd. 4 édition). Paris : Armand Colin.

Dubet, F. (2002). Le déclin de l'institution. Paris : Seuil.

Dufour, A. (1998). Les enjeux de l'enseignement de la sociologie dans une école d'ingénieur: le cas de l'Institut Supérieur d'Agriculture de Rhône-Alpes de 1968 à 1994.Thèse de doctorat en sciences de l'éducation. Lyon : Université de Lyon 2.

Durand, G. (2005). Introduction générale à la bioéthique. Histoire, concepts, outils. Québec : Fides.

Durkheim, E. (1967 [1893]). De la division du travail social (éd. 8 édition). Paris : Presses

Universitaires de France.

Ecole nationale d'ingénieurs de Brest (ENIB). (2014). L'éthique en pratique à l'ENIB. Brest : ENIB.

Ellul, J. (1954). La technique ou l'enjeu du siècle. Paris : Armand Colin.

Etchegoyen, A. (1991). La valse des éthiques. Paris : Bourin.

Faucheux, M., et Forest, J. (2007). Les recherches en sciences humaines et sociales dans les écoles

d'ingénieurs. Paris : Pétra.

Forquin, J.-C. (2008). Sociologie du curriculum. Rennes : Presses Universitaires de Rennes. 
Galiacy, D., et Havelange, V. (1983). Les enseignements de la philosophie dans les formations d'ingénieurs en France, en Belgique et à l'Université de Technologie de Compiègne. Compiègne : UTC.

Goffi, J.-Y. (1996). La philosophie de la technique. Paris : Presse Universitaire de France. Goujon, P., and Hériard Dubreuil, B. (2001). Ethics and Technology. Leuven : Peters.

Harris, C. E., Pritchard, M. S., and Rabins, M. J. (1995). Engineering Ethics : concept and cases. Belmont : Wadsworth Publishing Company.

Hériard Dubreuil, B. (1997). Imaginaire technique et éthique sociale. Bruxelles : De Boeck.

Huning, A., et Mitcham, C. (1993). The Historical and Philosophical Development of Engineering Ethics in Germany. Technology and Society, 15, 427-439. DOI : 10.1016/0160-791X(93)90014-F

Hunt, L. (1995 [1991]). L'affaire Paperclip. La récupération des scientifiques nazis par les américains 1945-1990. Paris : Stock.

Isambert, F.-A., Ladrière, P., et Terrenoire, J.-P. (1978). Pour une sociologie de l'éthique. Revue Française de Sociologie, 19(3), 323-339. http://www.persee.fr/doc/

rfsoc_0035-2969_1978_num_19_3_6911

Jarausch, K. H. (1990). The Unfree Professions : German Lawyers, Teachers and Engineers 1900-1950. Oxford : Oxford University Press.

Jonas, H. (1995[1979]). Le principe responsabilité. Une éthique pour la civilisation technologique. Paris : Flammarion.

Kahn, P. (2006). Réflexions générales sur l'éthique professionnelle enseignante. Recherche et Formation, 55, 105-116.

Larochelle, G. (2013). Le Québec sous l'emprise de la corruption. Cités, 1(53), 159-164. DOI : 10.3917/cite.053.0159

Layton, E. (1971). Revolt of the Engineering Profession. Social Responsibility and the American Engineering Profession. Baltimore et London : John Hopkins University.

Lazuech, G. (1998). La formation des cadres et ingénieurs : entre savoir et savoir-être. Contribution à une sociologie du processus d'internationalisation. Thèse de doctorat en Sociologie, Nantes : Université de Nantes.

Legault, G. A. (2007). Ethique et complexité dans le domaine du génie : Quelle formation humaniste retenir? Dans B. Feltz, P. Goujon, B. Hériard Dubreuil, S. Lavelle, et W. Lesch, Ethique, complexité et démocratie (pp. 237-252). Louvain-la-neuve : Academia-Bruylant.

Legault, G.A., Begin, L., et Racine, L. (1991). Ethique et ingénierie. Montréal : Mc Graw-Hill.

Lemaître, D. (2001). Formation humaine dans les écoles d'ingénieurs. Etude des conceptions contemporaines (anthropologique, éthique et culturelle). Thèse de doctorat en sciences de l'éducation. Nantes : Université de Nantes.

Lemaître, D. (2003). La formation humaine des ingénieurs. Paris : PUF.

Lemaître, D. (2011). Professionnalisation et modèles professionnels dans les grandes écoles françaises. Recherche et Formation, 66, 93-106. DOI : 10.4000/rechercheformation.1154.

Lipovetsky, G. (1992). Le crépuscule du devoir. L'éthique indolore des nouveaux temps démocratiques. Paris : Gallimard.

Martin, M., et Schinzinger, R. (1983). Ethics in Engineering. New-York : McGraw-Hill. 
Mitcham, C. (1993).Teaching Engineering Ethics : Resources available in North Amercia. Bulletin de la Société pour la philosophie des techniques, 5, 24-31.

Mitcham, C. (2003). Professional Idealism among Scientists and Engineers : a Neglected Tradition in STS Studies. Technology in Society, 25, 249-262. DOI : 10.1016/S0160-791X(03)00019-8

Mitcham, C. (2015). Ethics is not Enough. From Professionalism to the Political Philosophy of Engineering. Dans S. Sundar Sethy, Contemporary Issues in Engineering Ethics (pp. 48-80). Hershey, USA : IGI Global.

Monceau, G. (2006). Ethique et idéologie professionnelle. Recherche et formation, 52, 55-70. DOI : 10.4000/rechercheformation.1216

Parsons, T. (1939). The Professions and the Social Structure. (O. U. Press, Éd.) Social Forces, 17(4), 457-467.

Parsons, T. (1968). Profession. Dans Encyclopedia of the Social Science (Vol. 12, pp. 536-547). NewYork : Collier-McMillan.

Patenaude, J., et Legault, G. A. (1996). Enjeux de l'éthique professionnelle. Sainte Foy : Presses universitaires du Québec.

Prévost, P., et Jouffray, A. (2013). Le développement durable dans les formations d'ingénieurs en France : comment se situer entre la formation professionnelle et les « éducation à... ». Les cahiers du CERFEE, 33. DOI : $10.4000 /$ edso. 104

Racine, J., Légault, G.A., et Begin, L. (1991). Ethique et ingénierie. Montréal : McGraw-Hill.

Racine, L. (1990). Déontologie et éthique de l'ingénierie. Ethica, 2(1), 109-122.

Roby, C. (2014). Place et fonction des SHS dans les écoles d'ingénieurs en France : Etats des lieux, enjeux et perspectives épistémiques. Thèse de doctorat en sciences de l'éducation. Rennes : Université de Rennes.

Roby, C., et Albero, B. (2013). Formations des ingénieurs et SHS : les apports de deux thèses pionnières. TransFormations : Recherches en éducation des adultes (pp. 143-160). Lille : Trigone.

Ropohl, G. (2001).The Ethics of Technology. Dans P. Goujon, et B. Hériard Dubreuil,Technology and Ethics.The Quest of Responsible Engineering (pp. 45-66). Leuven : Peeters.

Schaub, J. H., et Pavlovic, K. (1983). Engineering Professionalism and Ethics. Malabar : Robert Krieger Publishing.

Sellier, A. (1998). Histoire du camp de Dora. Paris : La Découverte.

Sonntag, M., Lemaître, D., Fraysse, B., Becerril, R., et Oget, R. (2008). Les questions de formation dans les écoles d'ingénieurs. Un débat reconnu, une place pour la recherche ? Recherche et éducation, 1, 121-144. URL : http://rechercheseducations.revues.org/448

Terrenoire, J.-P. (1991). Sociologie de l'éthique professionnelle. Contribution à la réflexion théorique. Société contemporaine, 7, 7-33. http://www.persee.fr/doc/ socco_1150-1944_1991_num_7_1_1007

Thiel, M.-J. (2014). Questionnement autour de l'enseignement de l'éthique. Dans E. RudeAntoine, et al., Un état des lieux de la recherche et de l'enseignement en éthique (pp. 173-188). Paris : l'Harmattan.

Triffaux, J.-P. (1997). La culture générale et humaine de l'ingénieur : un facteur d'innovation. Economie et humanisme, 340, 54-55. 
Ullern-Weité, I. (2007). Enseigner l'éthique comme expertise ou former les personnes au jugement éthique de l'action? Question didactique. Nouvelle revue de psychosociologie, 1(3), 149-166. DOI : 10.3917/nrp.003.0149

Valadier, P. (1991). Inévitable morale. Seuil/Esprit.

Van de Poel, I. R., Zandvoort, H., et Brumsen, M. (2001). Ethics and Engineering Courses at Delft university of Technology : Contents, Educations Setup and Experiences. Science and Engineering Ethics, 7 (2), 267-282.

Van de Poel, I., et Royakkers, L. (2011). Ethics,Technology and Engineering : an Introduction. WileyBlackwell.

Vinck, D. (2007). La médiation sociologique, entre éthique et pratique, la connaissance. Dans B. Feltz, P. Goujon, B. Hériard Dubreuil, S. Lavelle, et W. Lesch, Ethique, technique et démocratie (pp. 253-270). Louvain-la-Neuve, Belgique : Academia-Bruyland.

Vinck, D. (2016). Les Sciences Studies : de la marginalité thématique à la refondation de la discipline. SociologieS, Dossiers, Sociétés en mouvement, sociologie en changement. URL : http:// sociologies.revues.org/5248

\section{NOTES}

1. CEFI, créé en 1975 par les Ministères de l'industrie et des universités

\section{RÉSUMÉS}

Les formations à l'éthique en écoles d'ingénieurs existent en France depuis une vingtaine d'années, mais elles restent plus rares que dans d'autres pays et que dans les formations médicales françaises. Cet article propose d'identifier les leviers d'action qui ont permis d'institutionnaliser l'éthique de l'ingénierie dans d'autres pays. Une comparaison avec le champ de la médecine permettra également d'identifier quelques obstacles propres au domaine de l'ingénierie dans le contexte français.

Courses in engineering ethics have existed in France for twenty years, but they are less common than in other countries and in medical education. In this paper, the author brings to light some reasons why engineering ethics did not developp in France as a field for research and education the way it did in other countries and it did to a certain point in the medecine in France.

\section{INDEX}

Mots-clés : éthique, ingénierie, médecine, professionnalisation, professionnalité, interdisciplinarité

Keywords : ethics, engineering, medicine, professionalization, professionalism, interdisciplinary 
AUTEUR

CHRISTELLE DIDIER

Université de Lille, CIREL 